\title{
Local Structure Around Te in Heavily Doped GaAs:Te using X-Ray Absorption Fine Structure
}

\author{
A. Pietnoczka ${ }^{a *}$, R. Bacewicz ${ }^{a}$, T. Slupinski ${ }^{b}$, J. Antonowicz $^{a}$, Su-Huai Wei $^{c}$ \\ ${ }^{a}$ Warsaw University of Technology, Faculty of Physics, Koszykowa 75, Warszawa 00-662, Poland \\ ${ }^{b}$ Warsaw University, Faculty of Physics, Hoża 69, Warszawa 00-681, Poland \\ ${ }^{c}$ National Renewable Energy Laboratory (NREL), 1617 Cole Blvd, Golden, CO 80401, United States
}

The annealing of heavily doped GaAs:Te can significantly change the free electron concentration in a reversible manner. These changes of electrical properties are accompanied by the structural changes of GaAs:Te solid solution. We used X-ray Absorption Fine Structure at K-edge of tellurium to determine local changes around Te atoms for different states of the GaAs:Te crystals caused by the annealing corresponding to different electron concentrations. The best EXAFS fit for the samples with high electron concentration was obtained for the substitutional $\mathrm{Te}_{\mathrm{As}}$ model with elongated $\mathrm{Te}-\mathrm{Ga}$ bonds (as compared to the As-Ga distance). For the samples in the low concentration state the best fit was for the pairs of Te atoms forming a rhombohedral symmetry double-DX centre, with the proportional admixture of the substitutional tellurium

PACS: 61.05.cj, 61.72.uj

\section{Introduction}

In heavily doped GaAs:Te reversible changes of the free electron concentration with the high temperatures annealing were observed since early sixties $[1,2]$. Tellurium doping of GaAs is used to provide n-type conductivity. Tetrahedrally coordinated Te substituting As is a shallow donor, and the electron concentration is usually close to the concentration of Te atoms. However, the annealing of heavily doped GaAs:Te can significantly change the free electron concentration in a reversible manner. Using the X-ray diffuse scattering technique, the reversible changes of the concentration were attributed to the structural changes in GaAs:Te solid solution [3, 4]. Various hypotheses were proposed to explain this deactivation mechanism in GaAs:Te. Fuller and Wolfstirn [1] suggested creation of "impurity molecules" which trap electrons. Some papers indicate gallium vacancy associated with the $\mathrm{Te}_{\mathrm{As}}$ quasisubstitutional defect as responsible for the observed compensation effect $[2,5,6]$. This complex was suggested on the grounds of Moessbauer experiments [5], scanning tunnelling microscopy [6], and other techniques. However, the $\mathrm{V}_{\mathrm{Ga}}-\mathrm{Te}_{\mathrm{As}}$ complex hypothesis does not explain the recovery of the electron concentration upon the high temperature annealing. Instead, the reversible changes of the concentration were attributed in $[3,4]$ to the creation of the impurity pairs which were revealed using the $\mathrm{X}$-ray diffuse scattering technique. We used X-ray Absorption Fine Structure at K-edge of tellurium to examine the local changes around Te for different states of GaAs:Te crystals corresponding to different electron concentrations.

* e-mail: pietnoczka@if.pw.edu.pl

\section{Experiment}

GaAs:Te crystal has been grown by Czochralski method. Details of samples used in this study are presented in Table I. Tellurium impurity concentrations were determined based on the impurity segregation phenomena at liquid-solid phase transition during crystal growth [7]. Free electron concentrations were determined using resistivity and Hall effect measurements, with the uncertainity of $5 \%$. The thermal treatments of GaAs:Te, were conducted in an evacuated sealed quartz glass ampoule with As vapour atmosphere (to prevent decomposition at the GaAs). For the H1 sample the free electron concentration is approximately equal to the tellurium atoms concentration (we call it a high electron concentration state). For the L1 and L2 samples free electron concentration is lowered by annealing to $88 \%$ and $21 \%$ of the value of tellurium atoms concentration, respectively. We refer to these samples as the low electron concentration state.

TABLE I

List of the samples used in this study.

\begin{tabular}{|c|c|c|c|c|}
\hline \multirow[t]{2}{*}{ Sample } & \multicolumn{2}{|c|}{ Annealing } & Tellurium & Electron \\
\hline & $\mathrm{T}\left[{ }^{\circ} \mathrm{C}\right]$ & time [h] & \multicolumn{2}{|c|}{ concentration $\left[\mathrm{cm}^{-3}\right]$} \\
\hline H1 & 1185 & 4 & $(1.7-1.8) \times 10^{19}$ & $1.7 \times 10^{19}$ \\
\hline L1 & 1050 & 39 & $(1.5-1.7) \times 10^{19}$ & $1.5 \times 10^{19}$ \\
\hline $\mathrm{L} 2$ & 800 & 288 & $(1.7-1.8) \times 10^{19}$ & $3.5 \times 10^{18}$ \\
\hline
\end{tabular}

EXAFS measurements at the Te K-edge have been carried out at the $\mathrm{X} 1$ beamline of the DORIS 3 ring in the HASYLAB. Due to the high dilution of Te in our crystals $([\mathrm{Te}] \cong 0.1 \%)$ multiple-scans were recorded using 
the fluorescence detection. Because of Te dilution selfabsorption effects could be neglected. A typical fluorescence measurement geometry was used with a detector axis perpendicular to the X-ray beam and the sample surface at 45 degree angle to the X-ray beam. In order to limit thermal damping of EXAFS oscillations we kept the samples at a temperature of $80 \mathrm{~K}$.

\section{Results and discussion}

The EXAFS data were extracted from the raw absorption data with the ATHENA program, and non-linear fitting was carried out using the ARTHEMIS program (both programs from the IFEFFIT package $[8,9]$ ). The FEFF 8 code [10] was used for the calculation of the photoelectron scattering amplitudes and phases necessary in EXAFS data fitting It represents the real-space multiplescattering (RSMS) calculations method with summation of contributions from all photoelectron scattering paths in atomic clusters.

The various models for different states of the GaAs:Te crystals corresponding to their electron concentrations were considered. It was reasonable to assume, that all $\mathrm{Te}$ atoms reside in $\mathrm{Te}_{\mathrm{As}}$ substitutional sites in the high electron concentration state. For samples with partially deactivated tellurium various models were analyzed. First, the model in which substitutional $\mathrm{Te}_{\mathrm{As}}$ forms a complex with a neighbouring gallium vacancy $\left(\mathrm{V}_{\mathrm{Ga}}-\mathrm{Te}_{\mathrm{As}}\right)$, this complex was identified as responsible for the annealing induced reduction of electron concentration in a number of papers $[2,5,6]$. For the low electron concentration state we also took into account some models of DX centers proposed for Te in GaAs [11, 12]. For the substitutional $\mathrm{Te}_{\mathrm{As}}$ and $\mathrm{V}_{\mathrm{Ga}}-\mathrm{Te}_{\mathrm{As}}$ complex models we used a cluster with a radius $10 \AA$ containing 167 atoms. Structural data of GaAs (the space group F$43 \mathrm{~m}, a=5.65325 \AA$ ) have been used. The model of double-DX centre includes a pair of Te atoms (the TeTe distance equal to $7.6 \AA$ ) along $\langle 110\rangle$ direction and additionally cation-cation bonded state (Fig. 1). Calculations of the centre formation energies using the density functional theory (VASP code $[13,14]$ ) indicate that such a defect complex is more stable than Te in the ideal zinc-blende structure. In the EXAFS calculation we used atomic positions obtained within that scheme.

EXAFS oscillations and their Fourier transforms for the samples studied are shown in the Fig. 2. The Fourier transforms were made in the k-range $\left(2.5-12.0 \AA^{-1}\right)$. Differences between plots are visible in the second and fourth shell peaks. For the annealing compensated state (low concentration state) the peak appears around $3.2 \AA$ and the fourth shell peak (around $4.2 \AA$ ) is much lower. It should be emphasized that the first shell EXAFS is almost the same for all plots. Moreover we cannot see differences in the first-neighbour-shell ordering: the FWHM value of the first peak is almost equal for all samples (0.51 $\AA$ on average). Our results indicate, that atomic distances in the first coordination shell stay practically unchanged upon the structural changes.

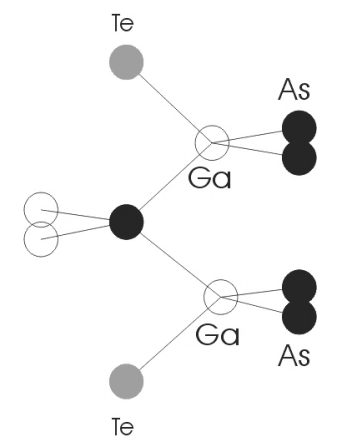

Fig. 1. Schematic atomic structure of the rhombohedral symmetry double-DX centre.

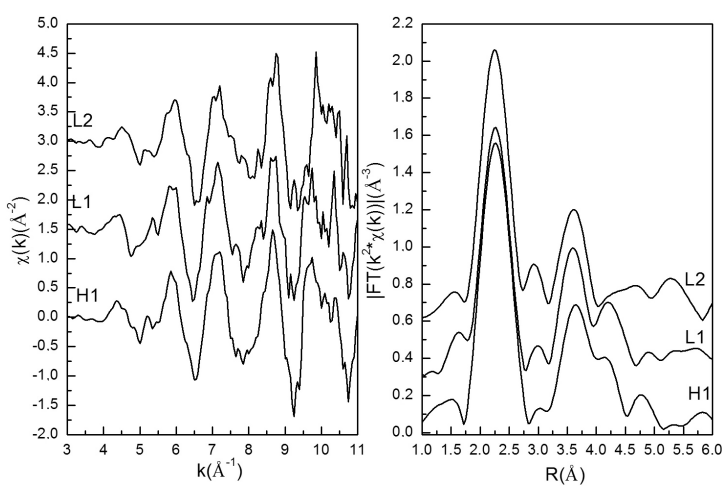

Fig. 2. EXAFS oscillations (multiplied by $k^{2}$ ) and their Fourier transforms for the crystals used in the study.

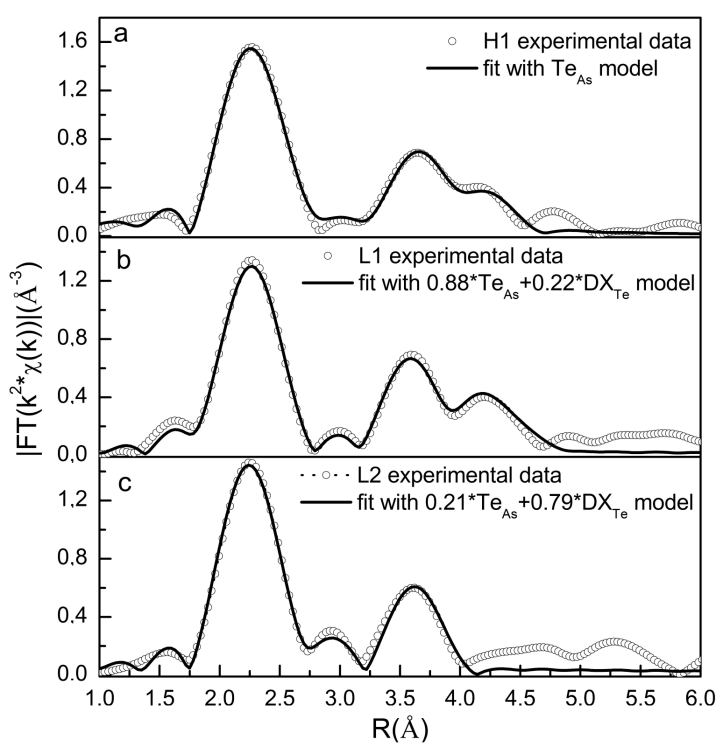

Fig. 3. Fourier-transformed $k^{2}$-weighted EXAFS with the best fits for the H1(a), L1 (b) and L2 (c) samples. 


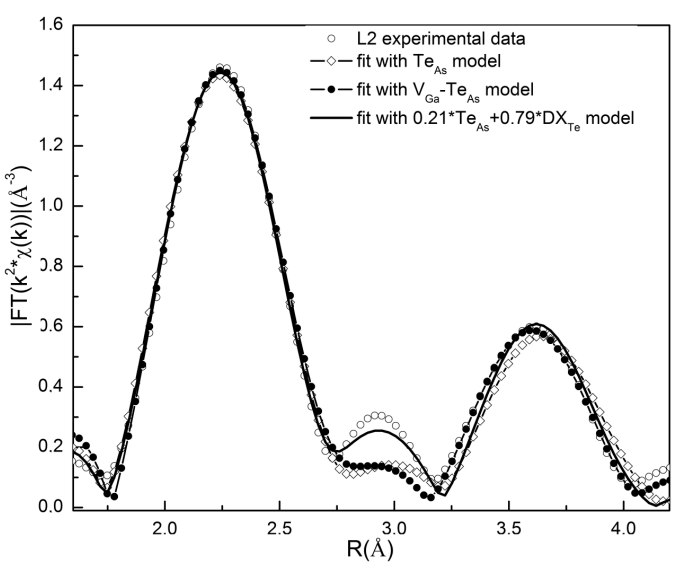

Fig. 4. A comparison of the fits for different models ( $\mathrm{Te}_{\mathrm{As}}, \mathrm{Te}_{\mathrm{As}}+\mathrm{DX}_{\mathrm{Te}}$ and $\left.\mathrm{V}_{\mathrm{Ga}}-\mathrm{Te}_{\mathrm{As}}\right)$ for the $\mathrm{L} 2$ sample.

The fitting of the EXAFS equation have been carried out in the R-space in the $1.8-5.0 \AA$ range for the $\mathrm{H} 1, \mathrm{~L} 1$ samples and in the 1.8-4.2 $\AA$ range for the L2 sample. This corresponds to three coordination shells around a Te site for the H1 and L2 samples and four coordination shells for the L1 sample. We kept the coordination numbers equal to the nominal values. For the substitutional $\mathrm{Te}_{\mathrm{As}}$ model: $4 \mathrm{Ga}$ atoms in the nearest-neighbour shell, 12 As atoms in the second-neighbour shell and 12 $\mathrm{Ga}$ atoms in the third-neighbour shell. For the model with 2 paired Te atoms forming rhombohedral symmetry double-DX centre: $3 \mathrm{Ga}$ atoms in the first-neighbour shell, $1 \mathrm{Ga}$ atom in the second-neighbour shell and $12 \mathrm{As}$ atoms in the third-neighbour shell. The passive electron reduction factor $\left(S_{0}^{2}\right)$ for Te was set to 1.0 for all fits. This value was obtained from the fit with the substitutional model to the $\mathrm{H} 1$ data. The fit quality was judged from the $\mathrm{R}$-factor value and reduced chi-square $\left(\chi_{\text {red }}^{2}\right)[8,9]$. The best fit parameters are presented in Table II. It contains values and uncertainty of interatomic distances, the Debye-Waller factors and the values of quality of the fit (R-factors and $\left.\chi_{\text {red }}^{2}\right)$.

TABLE II

The best EXAFS fit parameters for the substitutional $\mathrm{Te}_{\mathrm{As}}$ model and for the model assuming substitutional $\mathrm{Te}_{\mathrm{As}}$ and double-DX centre model. Both: $\chi_{\text {red }}^{2}$ and R-factor are given as the measures of the fitting quality. The values fixed during the fitting are underlined.

\begin{tabular}{|c|c|c|c|c|c|c|c|c|c|}
\hline \multirow[t]{2}{*}{ Sample } & \multirow[t]{2}{*}{$\chi_{\text {red }}^{2}$} & \multirow[t]{2}{*}{ R-factor } & \multirow[t]{2}{*}{ No. shell } & \multicolumn{3}{|c|}{ Model $\mathrm{Te}_{\mathrm{As}}$} & \multicolumn{3}{|c|}{ Model double-DX $\mathrm{T}_{\mathrm{Te}}$ centre } \\
\hline & & & & $\mathrm{R}(\AA)$ & $\mathrm{N}$ & $\sigma^{2}\left(\AA^{2}\right)$ & $\mathrm{R}(\AA)$ & $\mathrm{N}$ & $\sigma^{2}\left(\AA^{2}\right)$ \\
\hline \multirow{4}{*}{ H1 } & \multirow{4}{*}{0.81} & \multirow{4}{*}{0.011} & 1 & 2.66 & $4(\mathrm{Ga})$ & 0.002 & & & \\
\hline & & & 2 & & & & & & \\
\hline & & & 3 & 4.03 & $12(\mathrm{As})$ & 0.006 & & & \\
\hline & & & 4 & 4.72 & $12(\mathrm{Ga})$ & 0.010 & & & \\
\hline & & & \multicolumn{7}{|c|}{$0.88 * \mathrm{Te}_{\mathrm{As}}+0.12 * \mathrm{DX}_{\mathrm{Te}}$} \\
\hline \multirow{5}{*}{ L1 } & \multirow{4}{*}{0.72} & \multirow{4}{*}{0.008} & 1 & $\underline{2.65}$ & $4(\mathrm{Ga})$ & 0.004 & 2.66 & $3(\mathrm{Ga})$ & 0.004 \\
\hline & & & 2 & & & & 3.18 & $\overline{1(\mathrm{Ga})}$ & 0.003 \\
\hline & & & 3 & $\underline{4.03}$ & $12(\mathrm{As})$ & 0.007 & 4.07 & $\overline{12(\mathrm{As})}$ & 0.007 \\
\hline & & & 4 & $\underline{4.69}$ & $12(\mathrm{Ga})$ & 0.008 & & & \\
\hline & & & \multicolumn{7}{|c|}{$0.21 * \mathrm{Te}_{\mathrm{As}}+0.79 * \mathrm{DX}_{\mathrm{Te}}$} \\
\hline \multirow{3}{*}{ L2 } & \multirow{3}{*}{1.11} & \multirow{3}{*}{0.008} & 1 & $\underline{2.63}$ & $4(\mathrm{Ga})$ & 0.002 & 2.63 & $3(\mathrm{Ga})$ & 0.002 \\
\hline & & & 2 & & & & 3.18 & $\overline{1(\mathrm{Ga})}$ & 0.009 \\
\hline & & & 3 & $\underline{4.02}$ & $12(\mathrm{As})$ & 0.010 & 4.02 & $\overline{12(\mathrm{As})}$ & 0.010 \\
\hline \multicolumn{4}{|c|}{ uncertainity } & \pm 0.01 & & \pm 0.0005 & \pm 0.01 & & \pm 0.0005 \\
\hline
\end{tabular}

For the $\mathrm{H} 1$ sample the substitutional $\mathrm{Te}_{\mathrm{As}}$ model fits perfectly the EXAFS data (Fig. 3a), with dilatation of the nearest-neighbour distance due to the difference in atomic radii of Te and $\mathrm{As}$ (from $2.43 \AA$ for the $\mathrm{As}_{\mathrm{s}}-\mathrm{Ga}$ distance to $2.66 \AA$ for the Te-Ga bond length). Surprisingly, no significant increase in the interatomic distances is noted for the second- and third-neighbour shells.

The best EXAFS fits for the L1 and L2 samples were obtained with models of a mixed: substitutional
+ double-DX tellurium. Since electron concentration for those samples is $88 \%$ and $21 \%$ of the Te concentration we assumed that exactly this fraction of Te atoms reside in isolated substitutional sites and the rest are in the paired Te DX states.

For the model with 2 paired Te atoms forming rhombohedral symmetry double-DX centre, the theory predicts a dispersion of the bond lengths: $2.61-2.62 \AA$ for the first coordination shell and 3.90-4.13 $\AA$ for the third coordi- 
nation shell. In the fits we have to equalize distances for those coordination shells because the phase dispersion of the corresponding paths leads to vanishing of the EXAFS oscillation and abnormally low or even negative Debye-Waller factors for these shells.

In order to limit the number of fitting parameters for the mixed Te occupation model (substitutional + doubleDX tellurium) we fixed interatomic distances for the $\mathrm{Te}_{\mathrm{As}}$ model. These values are known from the fits with the substitutional model. We also used the same values of mean-square atomic displacements for respective shells for both models. In this way we had in total 7 fitting variables for L1 and 6 for L2. For both samples we used: 3 interatomic distances and 3 mean-square atomic displacements $\left(\sigma^{2}\right)$ for three coordination shells and for L1 it was one additional parameter $\sigma_{4}^{2}$ for fourth shell. Good results of fittings indicate that the assumed model involving pairs of Te atoms with additionally cation-cation bonded state describes correctly the local structure around the atom of tellurium. For those fits, we observed the dilatation of nearest-neighbor distance just like in the $\mathrm{H} 1$ sample. Results of fitting are presented in Fig. 3b,c.

For the low state samples there were also performed fits with models of $\mathrm{V}_{\mathrm{Ga}}-\mathrm{Te}_{\mathrm{As}}$ complex. In Fig. 4 the results of fitting for various considered models: $\mathrm{Te}_{\mathrm{As}}, \mathrm{Te}_{\mathrm{As}}+\mathrm{DX}_{\mathrm{Te}}$ and $\mathrm{V}_{\mathrm{Ga}}-\mathrm{Te}_{\mathrm{As}}$ are showed. For those fits the values of quality of the fit R-factor equals 0.026, 0.008 and 0.017, respectively. For the $\mathrm{V}_{\mathrm{Ga}}-\mathrm{Te}_{\mathrm{As}}$ model, the quality of the fit would be sufficient to treat it as good $(\mathrm{R}-$ factors $=$ 0.017 ) but the impossibility of reproducing the second peak (around $3.2 \AA$ ) proves the examined model to be incorrect (Fig. 4).

\section{Conclusions}

The aim of this study was to relate the local changes around Te atoms to different electron concentrations. For the H1 sample with high electron concentration the substitutional $\mathrm{Te}_{\mathrm{As}}$ model fits perfectly the EXAFS data, with elongation of the nearest neighbor distance, i. e. from $2.43 \AA$ for the As-Ga distance to $2.64 \AA$ for the Te$\mathrm{Ga}$ bond length. The dilatation of the nearest-neighbor distance is also observed for the samples in the low elec- tron concentration state. However, no significant increase in the atomic distances is noted for the further neighbor shells for all the samples. The model with 2 paired Te atoms forming rhombohedral symmetry double-DX centre gives good agreement with EXAFS data for the samples with partially deactivated tellurium. It agrees with the results of the $\mathrm{X}$-ray diffuse scattering measurements $[3,4]$, which indicate Te atoms pairs as responsible for the reduction of electron concentration.

\section{Acknowledgements}

This work was supported by the Polish State Committee for Scientific Research, Project No. IP2010 031370.

\section{References}

[1] C.S. Fuller, K.B. Wolfstirn, J. Appl. Phys. 34, 2287 (1963).

[2] C.J. Hwang, J. Appl. Phys. 40, 4591 (1969).

[3] J. Borowski, J. Gronkowski, E. Rohozińska, T. Słupiński, J. Phys. D-Appl. Phys. 31, 1883, (1998).

[4] T. Słupiński, E. Zielińska-Rohozińska, Thin Solid Films 367, 221 (2000).

[5] K. Wuys, G. Langouche, M. Van Rossum, R.E. Silverans, Phys. Rev. B 45, 6297 (1992).

[6] J. Gebauer, E.R. Weber, N.D. Jaeger, K. Urban, Ph. Ebert Appl. Phys. Lett. 82, 2059 (2003).

[7] W.G. Pfann, Solid State Physics 4, 423 (1957).

[8] M. Newville, B. Ravel, D. Haskel, J.J. Rehr, E.A. Stern, Y. Yacoby, Physica B 208/209, 154 (1995).

[9] E.A. Stern, M. Newville, B. Ravel, Y. Yacoby, D. Haskel, Physica B 208/209, 117 (1995).

[10] L. Ankundinov, B. Ravel, J.J. Rehr, S.D. Conradson, Phys. Rev. B 58, 756 (1998).

[11] D.J. Chadi, Phys. Rev. B 46, 6777 (1992).

[12] C.H. Park, D.J. Chadi, Phys. Rev. B 54, 14246 (1996).

[13] G. Kresse, J. Furthmüller, Comput. Mat. Sci. 6, 15 (1996).

[14] P.E. Blöchl, Phys. Rev. B 50, 17953 (1994). 